\title{
A RATIONAL NORMAL FORM FOR CERTAIN QUARTICS*
}

BY RAYMOND GARVER

A quartic equation with rational coefficients, irreducible in the field $R$ of rational numbers, will have for its Galois group (for the field $R$ ) one of the following:

I. The symmetric group of order 24 .

II. The alternating group of order 12 .

III. A group of order 8 such as the one whose substitutions are 1, (12), (34), (13)(24), (12)(34), (14)(23), (1324), (1423).

IV. A cyclic group of order 4 such as 1, (1324), (12)(34), (1423).

V. The 4-group 1, (12)(34), (13)(24), (14)(23).

It is the purpose of this note to give what seems to be a more direct proof of a theorem of Wiman : $\dagger$

Theorem. Any rational quartic, irreducible in $R$, whose Galois group for $R$ is either III, IV, or V above, can be transformed by a rational Tschirnhausen transformation into the form $y^{4}+p y^{2}+q=0$, where $p$ and $q$ are rational.

It will then follow as a corollary that $p$ and $q$ can be made integral; this can be accomplished by a simple additional transformation.

We may consider the given quartic in the reduced form

$$
x^{4}+a_{2} x^{2}+a_{3} x+a_{4}=0, \quad\left(a_{1}=s_{1}=0, a_{3} \neq 0\right) .
$$

Apply the quadratic transformation

$$
y=x^{2}+k_{1} x+k_{2} .
$$

Now the transformed equation will lack its second and fourth terms provided $\sum y=0, \sum y^{3}=0 . \ddagger$ The first of these equa-

* Presented to the Society, October 29; 1927.

$\dagger$ Arkiv för Matematik, Astronomi, och Fysik, vol. 3 (1906-7), No. 28.

$\ddagger$ The summations extend over the 4 roots of (1). 
tions, by (2), will be satisfied if $k_{2}=a_{2} / 2$. To obtain $\sum y^{3}$ we cube both sides of (2), sum over the four roots of (1), and use Newton's relations applied to (1), namely

$$
\left\{\begin{array}{l}
s_{2}=-2 a_{2}, \quad s_{3}=-3 a_{3}, \quad s_{4}=2 a_{2}^{2}-4 a_{4}, \\
s_{5}=5 a_{2} a_{3}, \quad s_{6}=6 a_{2} a_{4}-2 a_{2}{ }^{3}+3 a_{3}^{2} .
\end{array}\right.
$$

The equation $\sum y^{3}=0$ then takes the form

$$
a_{3} k_{1}^{3}-\left(a_{2}^{2}-4 a_{4}\right) k_{1}^{2}-2 a_{2} a_{3} k_{1}-a_{3}^{2}=0 .
$$

Now it is known* that if $x_{1}, x_{2}, x_{3}, x_{4}$ denote the roots of (1), then $y_{1}=x_{1} x_{2}+x_{3} x_{4}, y_{2}=x_{1} x_{3}+x_{2} x_{4}, y_{3}=x_{1} x_{4}+x_{2} x_{3}$ are the roots of the resolvent cubic

$$
y^{3}-a_{2} y^{2}-4 a_{4} y+4 a_{2} a_{4}-a_{3}^{2}=0 .
$$

And if the group of (1) is III, IV, or V, equation (5) will have at least one rational root. In this case (4) will also have a rational root, since the roots of (4) can be expressed rationally in terms of those of (5). It is easily verified that they are

$$
\frac{-a_{3}}{a_{2}-y_{1}}, \quad \frac{-a_{3}}{a_{2}-y_{2}}, \quad \frac{-a_{3}}{a_{2}-y_{3}} .
$$

These expressions for the roots of (4) were not, however, by any means obvious; the problem was to exhibit them in some form so that it would be possible to show that (4) has a rational root provided the group of (1) is III, IV, or V. For using this rational value of $k_{1}$, together with $k_{2}=a_{2} / 2$, we have exhibited the rational transformation which leads to the result of the theorem.

Bucht $\dagger$ has pointed out, and it is not difficult to show that if the group of (1) is $\mathrm{V}$, the $q$ of the transformed equation will be not only rational, but the square of a rational number.

The UNIVERSITY OF ROCHESTER

* Dickson, Elementary Theory of Equations, 1914, p. 39.

$\dagger$ Arkiv för Matematik, Astronomi, och Fysik, vol. 6 (1910-11), No. 30. 